\title{
Serendipitous discovery of the unidentified extended TeV $\gamma$-ray source HESS J1303-631
}

\author{
F. Aharonian ${ }^{1}$, A. G. Akhperjanian ${ }^{2}$, K.-M. Aye ${ }^{3}$, A. R. Bazer-Bachi ${ }^{4}$, M. Beilicke $^{5}$, W. Benbow ${ }^{1}$, D. Berge ${ }^{1}$,
} P. Berghaus ${ }^{6, \star}$, K. Bernlöhr ${ }^{1,7}$, C. Boisson ${ }^{8}$, O. Bolz ${ }^{1}$, I. Braun ${ }^{1}$, F. Breitling ${ }^{7}$, A. M. Brown ${ }^{3}$, J. Bussons Gordo ${ }^{9}$, P. M. Chadwick ${ }^{3}$, L.-M. Chounet ${ }^{10}$, R. Cornils ${ }^{5}$, L. Costamante ${ }^{1,20}$, B. Degrange ${ }^{10}$, A. Djannati-Ataï ${ }^{6}$, L. O'C. Drury ${ }^{11}$, G. Dubus ${ }^{10}$, D. Emmanoulopoulos ${ }^{12}$, P. Espigat ${ }^{6}$, F. Feinstein 9 , P. Fleury ${ }^{10}$, G. Fontaine ${ }^{10}$, Y. Fuchs ${ }^{13}$, S. Funk ${ }^{1}$, Y. A. Gallant ${ }^{9}$, B. Giebels ${ }^{10}$, S. Gillessen ${ }^{1}$, J. F. Glicenstein ${ }^{14}$, P. Goret ${ }^{14}$, C. Hadjichristidis ${ }^{3}$, M. Hauser ${ }^{12}$, G. Heinzelmann ${ }^{5}$, G. Henri' ${ }^{13}$, G. Hermann ${ }^{1}$, J. A. Hinton ${ }^{1}$, W. Hofmann ${ }^{1}$, M. Holleran ${ }^{15}$, D. Horns ${ }^{1}$, O. C. de Jager ${ }^{15}$, B. Khélifi ${ }^{1}$, Nu. Komin ${ }^{7}$, A. Konopelko ${ }^{1,7}$, I. J. Latham ${ }^{3}$, R. Le Gallou ${ }^{3}$, A. Lemière 6 ,

M. Lemoine-Goumard ${ }^{10}$, N. Leroy ${ }^{10}$, T. Lohse ${ }^{7}$, O. Martineau-Huynh ${ }^{16}$, A. Marcowith ${ }^{4}$, C. Masterson ${ }^{1,20}$, T. J. L. McComb ${ }^{3}$, M. de Naurois ${ }^{16}$, S. J. Nolan ${ }^{3}$, A. Noutsos ${ }^{3}$, K. J. Orford ${ }^{3}$, J. L. Osborne ${ }^{3}$, M. Ouchrif ${ }^{16,20}$, M. Panter ${ }^{1}$, G. Pelletier ${ }^{13}$, S. Pita ${ }^{6}$, G. Pühlhofer ${ }^{1,12}$, M. Punch $^{6}$, B. C. Raubenheimer ${ }^{15}$, M. Raue ${ }^{5}$, J. Raux ${ }^{16}$, S. M. Rayner ${ }^{3}$, I. Redondo ${ }^{10,20, \star \star}$, A. Reimer ${ }^{17}$, O. Reimer ${ }^{17}$, J. Ripken ${ }^{5}$, L. Rob $^{18}$, L. Rolland ${ }^{16}$, G. Rowell ${ }^{1}$, V. Sahakian ${ }^{2}$, L. Saugé ${ }^{13}$, S. Schlenker ${ }^{7}$, R. Schlickeiser ${ }^{17}$, C. Schuster ${ }^{17}$, U. Schwanke ${ }^{7}$, M. Siewert ${ }^{17}$, H. Sol ${ }^{8}$, R. Steenkamp ${ }^{19}$, C. Stegmann ${ }^{7}$, J.-P. Tavernet ${ }^{16}$, R. Terrier ${ }^{6}$, C. G. Théoret ${ }^{6}$, M. Tluczykont ${ }^{10,20}$, D. J. van der Walt ${ }^{15}$, G. Vasileiadis ${ }^{9}$, C. Venter ${ }^{15}$, P. Vincent ${ }^{16}$, H. J. Völk ${ }^{1}$, and S. J. Wagner ${ }^{12}$

\footnotetext{
${ }^{1}$ Max-Planck-Institut für Kernphysik, Heidelberg, Germany ${ }^{2}$ Yerevan Physics Institute, Armenia ${ }^{3}$ University of Durham, Department of Physics, UK ${ }^{4}$ Centre d'Étude Spatiale des Rayonnements, CNRS/UPS, Toulouse, France 5 Universität Hamburg, Institut für Experimentalphysik, Germany, e-mail: matthias.beilicke@desy.de ${ }^{6}$ APC, Paris, France ${ }^{\star \star \star} 7$ Institut für Physik, Humboldt-Universität zu Berlin, Germany ${ }^{8}$ LUTH, UMR 8102 du CNRS, Observatoire de Paris, Section de Meudon, France ${ }^{9}$ Laboratoire de Physique Théorique et Astroparticules, IN2P3/CNRS, Université Montpellier II, France ${ }^{10}$ Laboratoire Leprince-Ringuet, IN2P3/CNRS, École Polytechnique, Palaiseau, France ${ }_{11}$ Dublin Institute for Advanced Studies, Ireland ${ }^{12}$ Landessternwarte, Königstuhl, Heidelberg, Germany ${ }^{13}$ Laboratoire d'Astrophysique de Grenoble, INSU/CNRS, Université Joseph Fourier, Grenoble, France ${ }^{14}$ DAPNIA/DSM/CEA, CE Saclay, Gif-sur-Yvette, France ${ }^{15}$ Unit for Space Physics, North-West University, Potchefstroom, South Africa ${ }^{16}$ Laboratoire de Physique Nucléaire et de Hautes Énergies, IN2P3/CNRS, Universités Paris VI \& VII, France ${ }^{17}$ Institut für Theoretische Physik, Lehrstuhl IV: Weltraum und Astrophysik, Ruhr-Universität Bochum, Germany ${ }^{18}$ Institute of Particle and Nuclear Physics, Charles University, Prague, Czech Republic ${ }^{19}$ University of Namibia, Windhoek, Namibia

${ }^{20}$ European Associated Laboratory for Gamma-Ray Astronomy, jointly supported by CNRS and MPG
}

Received 5 April 2005 / Accepted 9 May 2005

\begin{abstract}
The serendipitous discovery of an unidentified extended $\mathrm{TeV} \gamma$-ray source close to the galactic plane named HESS J1303-631 at a significance of 21 standard deviations is reported. The observations were performed between February and June 2004 with the HESS stereoscopic system of Cherenkov telescopes in Namibia. HESS J1303-631 was discovered roughly $0.6^{\circ}$ north of the binary system PSR B 1259-63/SS 2883, the target object of the initial observation campaign which was also detected at $\mathrm{TeV}$ energies in the same field of view. HESS J1303-631 is extended with a width of an assumed intrinsic Gaussian emission profile of $\sigma=(0.16 \pm 0.02)^{\circ}$ and the integral flux above $380 \mathrm{GeV}$ is compatible with constant emission over the entire observational period of $(17 \pm 3) \%$ of the Crab Nebula flux. The measured energy spectrum can be described by a power-law $\mathrm{d} N / \mathrm{d} E \sim E^{-\Gamma}$ with a photon index of $\Gamma=2.44 \pm 0.05_{\text {stat }} \pm 0.2_{\text {syst }}$. Up to now, no counterpart at other wavelengths is identified. Various possible $\mathrm{TeV}$ production scenarios are discussed.
\end{abstract}

Key words. gamma rays: observations - Galaxy: disk

* Université Libre de Bruxelles, Faculté des Sciences, Campus de la Plaine, CP 230, Boulevard du Triomphe, 1050 Bruxelles, Belgium.

$\star \star$ Now at Department of Physics and Astronomy, Univ. of Sheffield, The Hicks Building, Hounsfield Road, Sheffield S3 7RH, UK.

$\star \star \star$ UMR 7164 (CNRS, Université Paris VII, CEA, Observatoire de Paris), France.

\section{Introduction}

Cherenkov telescopes of ground based $\gamma$-ray astronomy achieve a very high sensitivity. Due to the limited field of view the pointing of the telescopes has in general to be decided on the basis of observations in other wavelengths. However, 

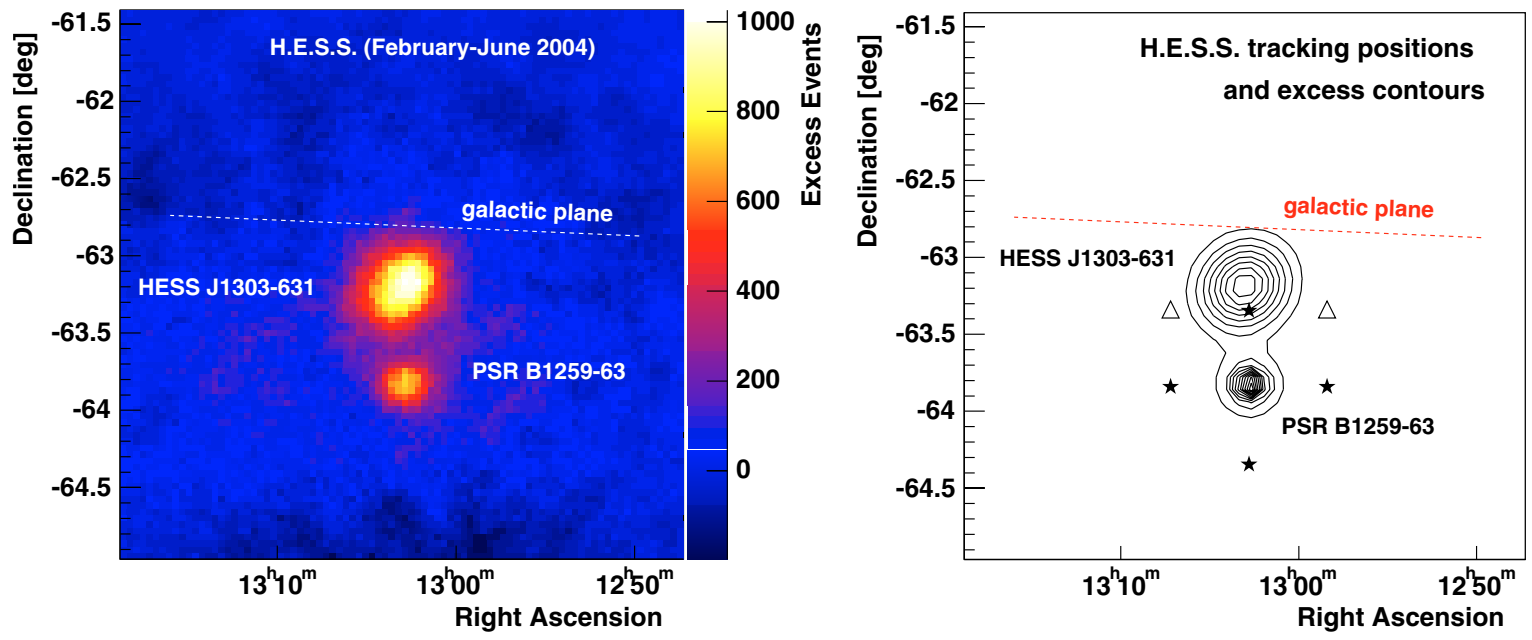

Fig. 1. Left: the sky map showing both TeV $\gamma$-ray sources: HESS J1303-631 and PSR B1259-63/SS 2883. The Galactic plane is also indicated. The number of events are integrated within a circle of $\Theta \leq 0.14^{\circ}$ for each of the correlated bins. Right: the function which was fitted to the (uncorrelated) excess sky map is drawn as contour lines. Also shown are the different HESS tracking positions: filled stars indicate the initial tracking positions (February-May) and the empty triangles the optimised positions which were used since May, 2004.

the comparatively large field of view of instruments such as HEGRA ( $4.3^{\circ}$ full angle) and HESS $\left(5^{\circ}\right.$ full angle) together with the stereoscopic observation mode allows nevertheless a scan of a part of the sky (Aharonian et al. 2002b, 2005b) and in particular allows a search for unknown sources in the field of view of individual pointings. In this way the first unidentified TeV $\gamma$-ray source TEV J2032+4130 has been discovered by HEGRA (Aharonian et al. 2002a, 2004a) in archival data and now, with the HESS telescopes, the second unidentified source HESS J1303-631 was discovered, followed by more unidentified sources found in the HESS galactic plane scan (Aharonian et al. 2005b). The HESS telescopes have originally been directed to search for $\mathrm{TeV} \gamma$-ray emission from the binary system PSR B1259-63/SS 2883 near periastron beginning in February 2004. This binary system has been detected at TeV energies (Beilicke et al. 2004) which is the subject of a parallel paper (see Aharonian et al. 2004c). Surprisingly, another TeV $\gamma$-ray source located at a position roughly $0.6^{\circ}$ north of the position of the binary system was discovered in the same field of view, given the name of HESS J1303-631 (see Fig. 1). The detection and basic features of this new source as well as a search for possible counterparts in other wavelengths and discussion of possible $\mathrm{TeV} \gamma$-ray production scenarios are reported in this paper.

\section{The HESS Cherenkov telescopes}

The HESS (High Energy Stereoscopic System) collaboration operates an array of four imaging atmospheric Cherenkov telescopes (IACTs) optimised for an energy range of $\gamma$-rays between $100 \mathrm{GeV}$ and $20 \mathrm{TeV}$. The telescopes are located in the Khomas Highland in Namibia $\left(23^{\circ} 16^{\prime} 18^{\prime \prime} \mathrm{S}, 16^{\circ} 30^{\prime} 1^{\prime \prime} \mathrm{E}\right)$ at a height of $1800 \mathrm{~m}$ above sea level. Each telescope has a $107 \mathrm{~m}^{2}$ tessellated mirror surface (Bernlöhr et al. 2003; Cornils et al. 2003) and is equipped with a 960 photomultiplier tube camera with a field of view diameter of $\sim 5^{\circ}$ (Vincent et al. 2003) which allows searches and studies of $\mathrm{TeV} \gamma$-ray sources in sky regions of more than $3^{\circ} \times 3^{\circ}$ per pointing. The telescopes are operated in a coincident mode (Funk et al. 2004) assuring that an event is always recorded by at least two of the four telescopes allowing for stereoscopic reconstruction of the shower parameters. More information about HESS can be found in Hinton (2004).

\section{Dataset}

The data were taken between February and June 2004 with the fully operational HESS IACT array. The average zenith angle of the observations was $42.7^{\circ}$ yielding an energy threshold of $E_{\text {thr }}=380 \mathrm{GeV}$, defined by the peak $\gamma$-ray detection rate of a Crab-like spectrum after event selection cuts. The observations were performed in the wobble mode, tracking a position shifted by $\pm 0.5^{\circ}$ in Declination or Right Ascension with respect to the nominal source position (in this case the PSR B1259-63/SS 2883 position of the initial observation campaign), allowing for an unbiased simultaneous background determination for not too extended sources. Following the detection of HESS J1303-631 in the PSR B1259-63/SS 2883 field of view, the telescope tracking positions were changed to new sky positions in May 2004, optimised for both sources by choosing the wobble modes with respect to a position located between PSR B1259-63/SS 2883 and HESS J1303-631. In the right hand panel of Fig. 1 the initial pointing positions (filled stars) as well as the new pointing positions (empty triangles) are shown.

The data were selected by standard quality criteria (stable weather and detector status) leaving $54.5 \mathrm{~h}$ of data $(48.6 \mathrm{~h} \mathrm{de}-$ tector life time) for the final analysis. For the data taken between February 26 and March 5, 2004 (7.6 h), one of the four telescopes was excluded from the analysis due to technical reasons concerning the event read-out of the telescope. The remaining data (March to June 2004) were analyzed using the full array of four telescopes. The raw data were subject to the standard calibration (Aharonian et al. 2004b) and Hillas parameterbased analysis (see Aharonian et al. 2005a for more details). 


\section{Analysis and results}

\subsection{Discovery of $\mathrm{TeV} \gamma$-ray emission from HESS J1303-631}

Shortly after the discovery of $\mathrm{TeV} \gamma$-ray emission from the binary system PSR B1259-63/SS 2883 the highly significant excess of the second source HESS J1303-631 was found in the data. The discovery sky map resolving both $\mathrm{TeV}$ sources is shown in the left hand panel of Fig. 1. The number of excess events was calculated using the ring background model in which the background is determined from a ring region with a radius $r>0.5^{\circ}$ centred around the putative source position. Both $\mathrm{TeV} \gamma$-ray source regions were excluded from the background region to avoid background contamination by real $\gamma$-ray events. In order to improve the angular resolution for the investigation of the position and extension, for the sky map at least three images instead of two were required per event.

\subsection{Consistency checks}

Since with the discovery of HESS J1303-631 for the first time in the HESS data and in $\mathrm{TeV} \gamma$-ray astronomy in general, two sources were found in the same field of view ${ }^{1}$ a wide range of consistency checks was applied and successfully passed underlining the celestial origin of the measured excess. For this purpose the data were divided into different subsets and the results (source strength, excess position, etc.) were compared. The subsets were chosen according to the different observation periods (from February to June, 2004) and the different telescope tracking positions (compare Fig. 1, right). Different cuts on the telescope multiplicity $m(m \geq 2, m=2, m \geq 3, m=3$ and $m=4$ ) for individual events as well as different cuts on the image amplitude were applied to the data. Also, individual telescopes in turn were excluded from the analysis. In all these checks the excess behaved as a genuine $\gamma$-ray source; more details can be found in Beilicke et al. (2005).

Finally, an important check concerns the distribution of the mean reduced scaled width (MRSW) parameter which is used for the $\gamma$-hadron separation (see Aharonian et al. 2005a). The $M R S W$ is obtained by averaging the reduced scaled widths of the individual images of an event. The reduced scaled width is defined as the difference (measured in standard deviations) between the measured and the expected width of an image. The expected width is obtained from Monte Carlo simulations for $\gamma$-ray showers, being parameterized as a function of the image amplitude, the shower impact parameter, and the zenith angle. The distribution of the MRSW parameter of the HESS J1303-631 excess is shown in Fig. 2 together with the distributions for $\gamma$-ray events from simulations and for hadronic events from a background measurement. The MRSW distribution of HESS J1303-631 is compatible with $\gamma$-ray events.

All results obtained from the different investigations are in good agreement. They were cross-checked with the template background model (Rowell 2003) and were also confirmed

\footnotetext{
${ }^{1}$ Meanwhile, two sources in one field of view have also been observed in other pointings with HESS, e.g. in the galactic center region and the galactic plane scan.
}

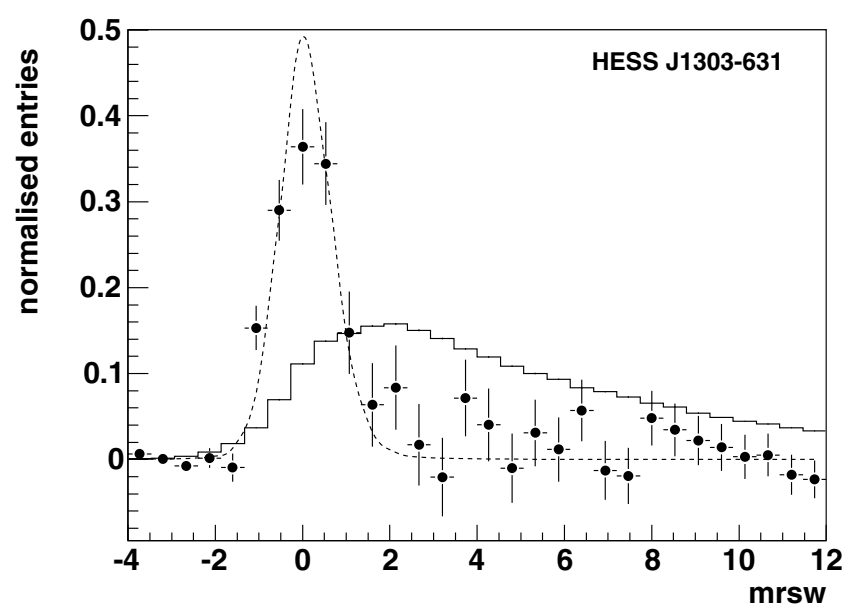

Fig. 2. Distribution of the mean reduced scaled width (MRSW) parameter (see text) for the HESS J1303-631 excess (data points). For comparison, the distributions for simulated $\gamma$-ray events (dashed line) and for hadronic events (histogram) are also shown. The excess measured from HESS J1303-631 is compatible with $\gamma$-ray events.

by an alternative model analysis technique (de Naurois et al. 2003). The celestial origin of the measured excess from HESS J1303-631 can therefore be considered as established.

\subsection{Excess and sky position}

To obtain the position of HESS J1303-631 a function describing the excess of the two sources in the field of view was fitted to the uncorrelated sky map which was generated with a cut on at least three images per event in order to obtain a better angular resolution. The excess of HESS J1303-631 was fitted by a 2D elliptical function (two-dimensional Gaussian function with a $\sigma$ for the Right Ascension and one for the Declination as well as a covariance). Simultaneously, the PSR B1259-63/SS 2883 position was fitted by the HESS point spread function (PSF) for $\mathrm{TeV} \gamma$-rays to avoid a systematic influence on the fit by excess events from PSR B1259-63 leaking into the HESS J1303631 region. The PSF can be described by a 2D double Gaussian function $a \cdot \exp \left(\frac{\Delta r^{2}}{2 \sigma_{1}^{2}}\right)+b \cdot \exp \left(\frac{\Delta r^{2}}{2 \sigma_{2}^{2}}\right)$ which was derived from Monte Carlo simulations. The fit range covers a region of $2^{\circ} \times 2^{\circ}$ centred at the HESS J1303-631 position. The parameters of the PSF $\left(\sigma_{1}, \sigma_{2}\right.$ and $\left.a / b\right)$ which was applied for the position of PSR B1259/SS 2883 were left free. The contour lines of the function are shown in the right hand panel of Fig. 1. The position of HESS J1303-631 was found to be $\alpha=13^{\mathrm{h}} 03^{\mathrm{m}} 0^{\mathrm{s}} .4 \pm 4.4$ and $\delta=-63^{\circ} 11^{\prime} 55^{\prime \prime} \pm 31^{\prime \prime}$ (J2000.0). The length and width of the fitted ellipse are within errors the same and clearly larger than that expected for a point source (see below). Therefore, the HESS J1303-631 excess is compatible with an extended and rotationally symmetric structure. The position of PSR B1259-63/SS 2883 obtained from the same fit was found to be $\alpha_{1259}=13^{\mathrm{h}} 02^{\mathrm{m}} 49.2 \pm 3.6$ and $\delta_{1259}=-63^{\circ} 50^{\prime} 2^{\prime \prime}$. $4 \pm 21^{\prime \prime}$. 1 which is in good agreement with its nominal position of $\alpha_{1259 \text {, nom }}=13^{\mathrm{h}} 02^{\mathrm{m}} 47.7$ and $\delta_{1259 \text {, nom }}=$ $-63^{\circ} 50^{\prime} 8^{\prime \prime} 8$. The width of the PSR B1259-63/SS 2883 excess was found to be compatible with the width of a pointsource (Aharonian et al. 2004c). Fixing the position of 


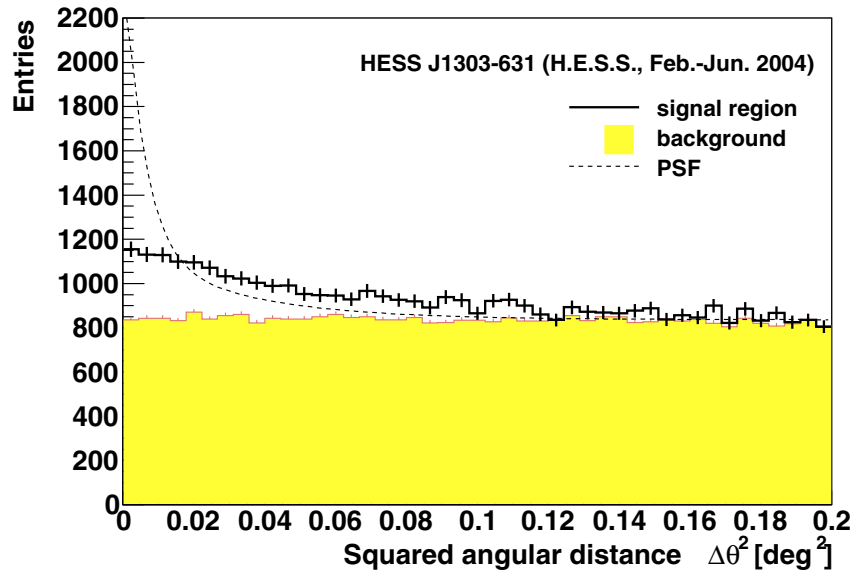

Fig. 3. Distribution of ON-source events (solid histogram) and normalized OFF-source events (filled histogram) vs. the squared angular distance $\Delta \Theta^{2}$ between the reconstructed shower direction and the object position. The expected ON-distribution for a point-like source (TeV point spread function, PSF) is indicated by the dashed line; it is normalized to give the same number of excess events as HESS J1303-631.

PSR B1259-63/SS 2883 in the fit to its nominal sky coordinates does not change the results for the HESS J1303-631 position and extension within statistics. The systematic pointing uncertainty of the HESS telescopes is estimated to be $\sim 20^{\prime \prime}$ for Right Ascension and Declination.

The distribution of the number of events in squared angular distance $\Delta \Theta^{2}$ measured between the reconstructed shower direction and the derived HESS J1303-631 position is shown in Fig. 3. The PSF of the HESS detector for TeV $\gamma$-rays is also shown (dotted line). A single Gaussian function describing the intrinsic source profile of HESS J1303-631 was folded with the PSF. The folded function was fitted to the excess distribution resulting in an intrinsic width of $\sigma_{\mathrm{HESS} \mathrm{J1303}}=(0.16 \pm 0.02)^{\circ}$ with a $\chi^{2} /$ d.o.f. $=21 / 42$.

Adjusting the angular cut to the derived extension $\left(\Delta \Theta^{2} \approx\right.$ $\Delta \Theta_{\text {pointsrc }}^{2}+\sigma_{\text {HESS J1303 }}^{2} \approx 0.05 \mathrm{deg}^{2}$ ) one obtains for the HESS J1303-631 position 2469 \pm 119 excess events corresponding to a significance of 21 standard deviations calculated following Li \& Ma (1983).

\subsection{Energy spectrum and light curve}

An energy spectrum was derived using the angular cut of $\Delta \Theta^{2}=0.05 \mathrm{deg}^{2}$. To obtain the full flux integrated over the whole emission region of HESS J1303-631 the flux normalisation was corrected for the derived source extension (assuming an intrinsic Gaussian emission profile). Since this correction depends on the exact - and possibly energy dependent - shape of the source, it introduces a systematic error. Together with the effects of atmospheric extinction variations and energy calibration of the detector the systematic error on the flux was estimated to be in the order of $\sim 30 \%$.

The spectrum is shown in Fig. 4. It was fitted by a powerlaw $\mathrm{d} N / \mathrm{d} E=N_{0} \cdot(E / 1 \mathrm{TeV})^{-\Gamma}$ with a resulting photon index of $\Gamma=2.44 \pm 0.05_{\text {stat }} \pm 0.2_{\text {syst }}$ and a normalization of $N_{0}=\left(4.3 \pm 0.3_{\text {stat }}\right) \times 10^{-12} \mathrm{~cm}^{-2} \mathrm{~s}^{-1} \mathrm{TeV}^{-1}$ with a $\chi^{2} /$ d.o.f.

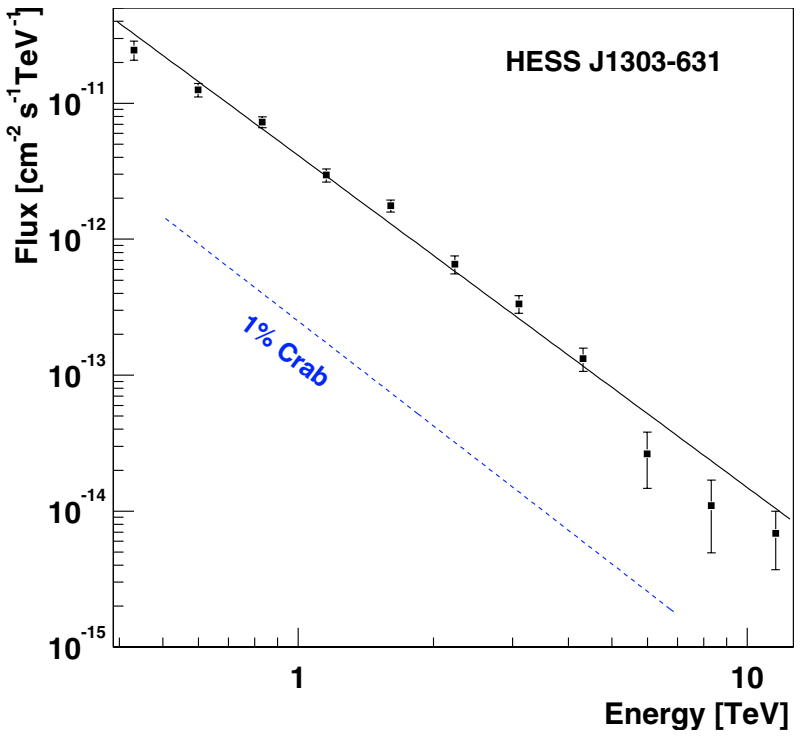

Fig. 4. The differential energy spectrum of HESS J1303-631. A fit to the data with a power law (solid line) is shown. The power-law corresponding to a spectrum of $1 \%$ of the Crab Nebula is also indicated (dashed line).

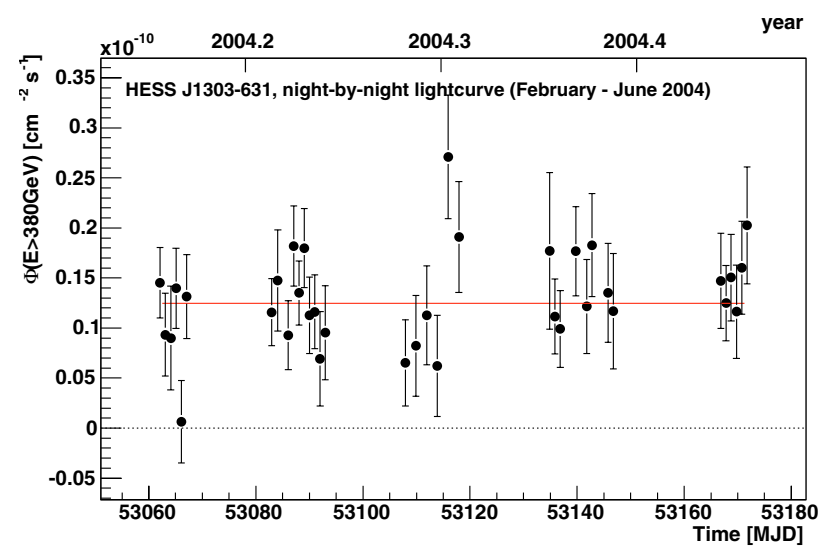

Fig. 5. The HESS J1303-631 light curve covering February until June, 2004. Shown is the integral flux $\Phi(E>380 \mathrm{GeV})$ vs. time in nightby-night bins. The fit of a constant function (solid line) results in a $\chi^{2} /$ d.o.f. of $35 / 35$ being compatible with constant emission.

of $27 / 9$. The integral flux above $380 \mathrm{GeV}$ was calculated to be $\Phi(E>380 \mathrm{GeV})=\left(1.2 \pm 0.2_{\text {stat }}\right) \times 10^{-11} \mathrm{~cm}^{-2} \mathrm{~s}^{-1}$ corresponding to $(17 \pm 3) \%$ of the flux of the Crab Nebula. Spectra obtained from the different tracking positions as well as from the different observation periods were found to be compatible within statistical errors. A fit of a curved spectral shape, e.g. with a power-law and a cut-off, yields a better $\chi^{2}$. However, at the present understanding of the systematic effects for extended sources observed with asymmetric wobble positions (comp. Fig. 1, right), we prefer not to quote numbers at this stage but to leave it to more detailed studies on morphology and spatiallyresolved energy spectra which are underway.

A light curve of the integral flux $\Phi(E>380 \mathrm{GeV})$ was derived on a night-by-night basis and is shown in Fig. 5. To obtain the integral flux, the count rates for each night were corrected using the effective areas and an assumed power-law as obtained 
from the overall differential energy spectrum. A fit of a constant function to the light curve results in a $\chi^{2} /$ d.o.f. of $35 / 35$ and therefore indicates constant emission from HESS J1303-631 during February until June, 2004.

\section{Search for possible counterparts}

\subsection{Search in catalogues}

As HESS J1303-631 is a new TeV source, a search for possible counterparts at other wavelengths was performed, using the VizieR service (Ochsenbein et al. 2000) at the Centre de Données astronomiques de Strasbourg. The closest EGRET $(>100 \mathrm{MeV})$ source, 3EG J1308-6112, has its centroid at an angular distance of $2.1^{\circ}\left(\mathrm{RA}=197^{\circ} .18, \mathrm{Dec}=-61.22\right)$, with a nominal $95 \%$ confidence radius of $0.71^{\circ}$ (Hartman et al. 1999). This source is flagged as possibly extended or multiple and confused, but examination of the actual position confidence contours confirms that it can be ruled out as a counterpart.

In the X-ray domain, the only catalogued sources found with VizieR within 30' of the centroid of HESS J1303-631 are from the Second ROSAT (0.24-2.0 keV) Source Catalogue of Pointed PSPC Observations (5 sources) or its WGACAT version (1 additional source). These ROSAT sources are listed in Table 1, and their positions are marked in Fig. 6 . The concentration of these sources to the South of HESS J1303-631 reflects the non-uniform sensitivity of PSPC observations across this field. Dedicated X-ray observations will be necessary to make a more complete search for counterparts.

As HESS J1303-631 is within the Galactic plane, optical or infrared sources are too numerous and have not been examined.

To search for counterparts in the radio domain, the $843 \mathrm{MHz}$ map of the region around HESS J1303-631 was obtained from SUMSS survey (Bock et al. 1999) and is shown as a grey-scale image in Fig. 6 . Those relatively bright sources within $30^{\prime}$ of HESS J1303-631 which are also identified in the $4.85 \mathrm{GHz}$ PMN (Parkes-MIT-NRAO) survey catalogue (Wright et al. 1994) are marked as circles or ellipses ${ }^{2}$ in Fig. 6 and are listed in Table 1 along with PMNJ1302-6321 which is the only catalogue source within the $1-\sigma$ source extension of HESS J1303-631, but is not detected in the $843 \mathrm{MHz}$ map. The region overlapping the centre of HESS J1303-631 appears devoid of conspicuous radio sources.

The nearest catalogued supernova remnant, Kes 17 (G 304.6+0.1), lies at a distance of 36' from HESS J1303-631 (Green 2004). VizieR also finds two HII regions within a 30' radius, G303.9-0.1 and G303.9-0.7 (Paladini et al. 2003). Finally, version 1.21 of the ATNF (Australia Telescope National Facility) Pulsar Catalogue ${ }^{3}$ (Manchester et al. 2005) lists five pulsars within a 30' radius around HESS J1303-631. The most compelling positional coincidences of radio sources with HESS J1303-631 appear to be with some of the pulsars, discussed below.

\footnotetext{
2 The objects marked PMN1 and PMN2 are consistent with point sources, and their diameter reflects the PMN beam size, while PMN3 and PMN4 are flagged as extended.

${ }^{3}$ http://www.atnf.csiro.au/research/pulsar/psrcat
}

Table 1. X-ray (ROSAT) and radio sources at a distance of less than $30^{\prime}$ from the centre of HESS J1303-631. The radio sources marked PMN are from the Parkes-MIT-NRAO catalogue, those marked $\mathrm{G}$ are $\mathrm{H}$ II regions, and those marked PSR are from the ATNF pulsar catalogue. Shown are the source name, the ID used in Fig. 6, the distance to the HESS J1303-631 centroid and the J2000.0 source coordinates.

\begin{tabular}{llrrr}
\hline \hline Source name & ID & dist. & RA & Dec \\
\hline \multicolumn{2}{c}{ X-ray sources (ROSAT) } & & & \\
1WGA J1303.8-6319 & WGA & $9.1^{\prime}$ & 130350.9 & -631906 \\
2RXP J130257.9-632750 & RXP1 & $15.9^{\prime}$ & 130257.9 & -632750 \\
2RXP J130540.5-631839 & RXP2 & $19.2^{\prime}$ & 130540.5 & -631839 \\
2RXP J130622.7-632210 & RXP3 & $24.9^{\prime}$ & 130622.7 & -632210 \\
2RXP J130200.4-633730 & RXP4 & $26.4^{\prime}$ & 130200.4 & -633730 \\
2RXP J130337.8-634045 & RXP5 & $29.1^{\prime}$ & 130337.8 & -634045 \\
radio sources & & & & \\
PMN J1302-6257 & PMN1 & $14.3^{\prime}$ & 130238.9 & -625748 \\
PMN J1300-6253 & PMN2 & $24.6^{\prime}$ & 130034.4 & -625343 \\
PMN J1303-6339 & PMN3 & $28.2^{\prime}$ & 130352.6 & -633929 \\
PMN J1259-6333 & PMN4 & $29.7^{\prime}$ & 125959.1 & -633337 \\
PMN J1302-6321 & PMN5 & $9.4^{\prime}$ & 130256.7 & -632120 \\
H II regions & & & & \\
G303.9-0.1 & G1 & $25.4^{\prime}$ & 125956.6 & -625722 \\
G303.9-0.7 & G2 & $28.9^{\prime}$ & 130007.3 & -633320 \\
pulsars & & & & \\
PSR J1302-6313 & PSR1 & $4.9^{\prime}$ & 130219.2 & -631329 \\
PSR J1303-6305 & PSR2 & $6.9^{\prime}$ & 130300.0 & -630501 \\
PSR J1301-6310 & PSR3 & $10.4^{\prime}$ & 130128.3 & -631041 \\
PSR J1301-6305 & PSR4 & $10.6^{\prime}$ & 130145.8 & -630534 \\
PSR J1305-6256 & PSR5 & $22.6^{\prime}$ & 130528.0 & -635639 \\
\hline
\end{tabular}

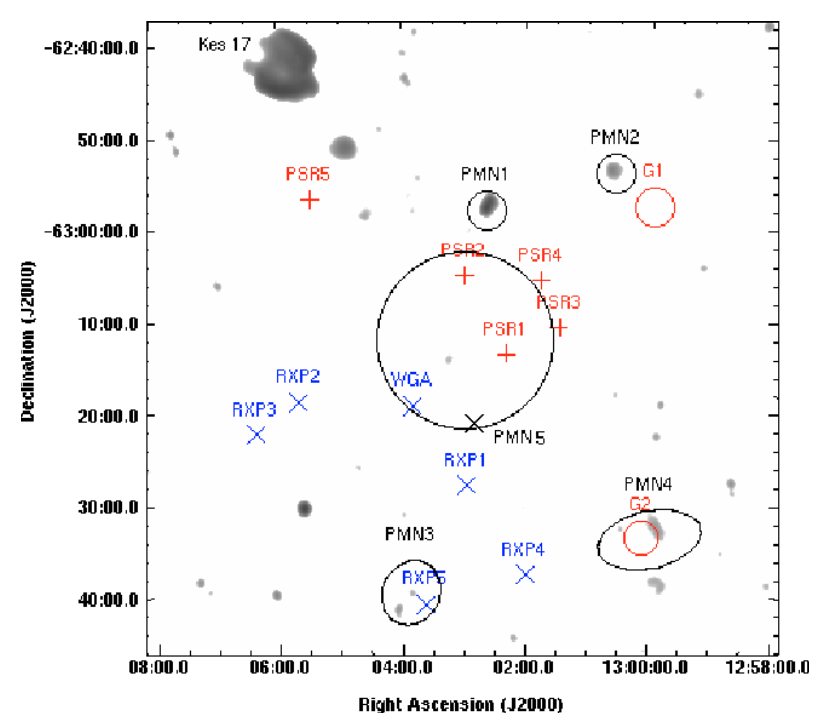

Fig. 6. $843 \mathrm{MHz}$ SUMSS radio map of the region around HESS J1303-631 (marked as the large circle of radius $0.16^{\circ}$ ). Also marked are the positions of the X-ray sources (WGA and RXP1-5) as well as the radio sources (PMN1-5, G1 and G2 for H II regions and PSR1-5 for pulsars) listed in Table 1. Circles or ellipses surrounding individual sources indicate the catalogued source extension (convolved with the relevant radio beam size).

\subsection{Possible pulsar wind nebula associations}

Pulsars can have associated very high energy (VHE) $\gamma$-ray emission from the nebulae of high-energy particles (mostly electrons and positrons) which they produce, as the wellknown example of the Crab Nebula shows. The $\mathrm{TeV}$ radiation is thought to arise from inverse Compton (IC) scattering of 
Table 2. Parameters of the pulsars possibly associated with HESS J1303-631. Listed are the pulsar name, its period $P$, estimated distance $D$, spindown age $\tau$ and flux $F_{\text {sd }}$ (see text).

\begin{tabular}{llrrr}
\hline \hline PSR name & $P(\mathrm{~s})$ & $D(\mathrm{kpc})$ & $\tau(\mathrm{kyr})$ & $F_{\text {sd }}\left(\mathrm{erg} \mathrm{cm}^{-2} \mathrm{~s}^{-1}\right)$ \\
\hline $\mathrm{J} 1302-6313$ & 0.968 & 28.06 & 2420 & $2.9 \times 10^{-15}$ \\
$\mathrm{~J} 1303-6305$ & 2.307 & 13.62 & 16800 & $3.1 \times 10^{-16}$ \\
$\mathrm{~J} 1301-6310$ & 0.664 & 2.06 & 186 & $1.5 \times 10^{-11}$ \\
$\mathrm{~J} 1301-6305$ & 0.185 & 15.84 & 11 & $5.6 \times 10^{-11}$ \\
$\mathrm{~J} 1305-6256$ & 0.478 & 30.00 & 3590 & $7.1 \times 10^{-15}$ \\
\hline
\end{tabular}

lower-energy ambient photons by these energetic electrons, which also emit synchrotron radiation at lower energies, typically between the infrared and X-ray bands. In the case of a relatively young (950 year old) pulsar like the Crab, the pulsar wind nebula (PWN) is fairly compact and appears point-like in VHE $\gamma$-rays, but in older pulsars the emission might be considerably more extended, due to the expansion of the PWN itself (e.g. van der Swaluw et al. 2001) or due to the escape of the high-energy particles out of the synchrotron-emitting PWN, as proposed by Aharonian et al. (1997).

To examine the possibility that HESS J1303-631 might be associated with such an extended nebula from one of the positionally compatible pulsars, the latters' energetics are now considered. The total available energy output of a pulsar is given by its spindown luminosity $\dot{E}$. It is believed that the major fraction of this energy is ultimately converted to accelerated particles, and the energetic electrons and positrons can radiate their energy away efficiently in the form of synchrotron and inverse Compton emission. In such a scenario the measured VHE energy flux from a source at the distance $D$ can be compared to $F_{\text {sd }} \equiv \dot{E} /\left(4 \pi D^{2}\right)$, whereby the conversion efficiency is expected to be much less than $100 \%$, i.e. the measured VHE flux is expected to be much less than $F_{\text {sd }}$.

Table 2 lists the relevant parameters for the five pulsars included in Table 1, as derived from version 1.21 of the ATNF Pulsar Catalogue. For comparison, the energy flux of HESS J1303-631 between 0.3 and $10 \mathrm{TeV}$ implied by its bestfit spectrum is $2.1 \times 10^{-11} \mathrm{erg} \mathrm{cm}^{-2} \mathrm{~s}^{-1}$. Some caveats should be kept in mind when comparing this number with the listed values of $F_{\mathrm{sd}}$ : uncertainties in the estimation of $D$ for individual pulsars can be significant; the comparison also implicitly assumes that the $\gamma$-ray emission is isotropic, but this is almost certainly a valid assumption for an extended source such as HESS J1303-631. Finally, delayed energy release of particles injected in the past, when the spindown luminosity was higher, could in principle increase the apparent efficiency; but this is unlikely to be a large effect in most evolutionary scenarios.

With this in mind, it can be seen that most of the pulsars listed in Table 2 can be ruled out as possible counterparts for HESS J1303-631 due to insufficient energetics. Only PSR J1301-6305 has enough energy to power the whole of the VHE emission, and even in this case the conversion efficiency would have to be unusually high.

\subsection{Molecular clouds, energetic stellar winds from Cen OB1, and SNR association}

If HESS J1303-631 is a result of cosmic ray interactions with ambient gas, one can draw the following conclusions: the fact that the spectrum of this source is significantly harder compared to the $E^{-2.7}$ diffuse (local) galactic cosmic ray spectrum, indicates that one does not see an interaction of this galactic cosmic ray component with regions of enhanced ambient target gas density, but rather of a source of cosmic rays, which is located within or near HESS J1303-631. If such a source is also in contact with a dense molecular cloud, the increased target density results in an increased rate for the production of VHE $\gamma$-rays as discussed by Aharonian et al. (1990).

Molecular clouds can provide the conditions for both type I and type II supernova events to occur, with the former resulting from accelerated accretion induced collapse of white dwarfs due to the increased gas density, and the latter resulting from the evolution of massive stars in star formation regions towards supernovae. Another possibility is that stellar winds from OB stellar associations, interacting with molecular clouds, may also be sources of $\gamma$-ray production, as suggested for TeV J2032+4130 by Aharonian et al. (2002b), or, such stellar winds may provide the required injection for further acceleration in a nearby SNR shell from the same or another nearby OB association (Montmerle 1979), called supernova-OB associations (or SNOBs). There is a possible correlation between SNOBs and unidentified $\gamma$-ray sources as found by Montmerle (1979) in the COS-B data, and in the EGRET data by Kaaret \& Cottam (1996).

As a first step the nature of molecular clouds in the direction of HESS J1303-631 is investigated, as reflected by CO transition line measurements, which gives (i) a kinematic distance based on galactic rotation curves (sometimes one will get more than one solution), and, (ii) the column density of gas corresponding to the region from where the $\mathrm{CO}$ emissions are seen.

It is possible to fit the observed spectral shape of HESS J1303-631 with a hadronic model. A hadron spectral index of 2.0 combined with an exponential cut-off at 20 to $50 \mathrm{TeV}$ would even account for the slight curvature apparent in the measured spectrum. To reproduce the observed TeV flux, one has to assume a total cosmic ray energy of $E_{\mathrm{CR}}=2$ to $5 \times 10^{49} d_{\mathrm{kpc}}^{2} / n_{1}$ erg, where $d_{\mathrm{kpc}}=d / 1 \mathrm{kpc}$ is the distance and $n_{1}=n / 1 \mathrm{~cm}^{-3}$ is the associated target density.

The procedure of deriving $n$ from the velocity integrated $\mathrm{CO}$ temperatures $\langle W(\mathrm{CO})\rangle=\int T \mathrm{~d} v$ is well-known, provided that a scale size for the cloud is specified (see e.g. Dame et al. 1993, and references therein). The deep CO survey of molecular clouds in the southern Milky Way by Bronfman et al. (1989) covers the $300^{\circ}$ to $348^{\circ}$ longitude and $-2^{\circ}$ to $+2^{\circ}$ galactic latitude ranges, with a spatial resolution of $0.125^{\circ}$, which is smaller than the resolved size of HESS J1303-631.

Extracting CO temperatures $T$ centred at $l=304.24^{\circ}$ and $b=-0.36^{\circ}$ (the centroid for HESS J1303-631) from this survey, results in three distinct spatial coincidences in velocity space. The merits of these will be discussed below. 
(i) It is unlikely that the Coalsack ( $v=-4$ to $-2 \mathrm{~km} \mathrm{~s}^{-1}$ ), at a distance of $\sim 175 \mathrm{pc}$ (Nyman et al. 1989), is a candidate environment for HESS J1303-631 based on two aspects: there is no evidence of star formation in this dark cloud (Nyman et al. 1989), and a bright counterpart should have been detectable at longer wavelengths, given the proximity of this cloud.

(ii) For the second $\mathrm{CO}$ solution the reader is refered to Fig. 11 of Bronfman (1989), who shows a clump in the velocity interval $\sim-30$ to $-15 \mathrm{~km} \mathrm{~s}^{-1}$ along the line-ofsight cutting through the centroid of the $\mathrm{TeV}$ source. The temperature was integrated along this velocity interval to give $W(\mathrm{CO})$, showing a plateau at the location of HESS J1303-631. This quantity, if averaged over the surface of the resolved $\mathrm{TeV}$ source, gives an average value of $\langle W(\mathrm{CO})\rangle \sim 15 \mathrm{~K} \mathrm{~km} \mathrm{~s}^{-1}$ for an average velocity of $v_{0} \sim-23 \mathrm{~km} \mathrm{~s}^{-1}$, which corresponds to a kinematic distance of either $d=2.1$ or $7.7 \mathrm{kpc}$, assuming the kinematic velocity model of Wouterloot \& Brand (1989), with the ambiguity arising from the galactic rotation curve. The $1^{\circ}$ cloud diameter converts to densities of $n \sim 56 \mathrm{~cm}^{-3}$ and $n \sim 15 \mathrm{~cm}^{-3}$ for the two distances, respectively, which translates to a total required cosmic ray energy of either $\sim 3 \times 10^{48}$ (for $d=2.1 \mathrm{kpc}$ ), or, $\sim 10^{50} \mathrm{erg}$ for $d=7.7 \mathrm{kpc}$. The size of the $\gamma$-ray source is determined by the size of the dense target (density profile) and the diffusion (propagation) length. The densities derived here will be required for the discussion below.

(iii) The third CO solution involves the detection of the giant molecular cloud (GMC) G303.9-0.4 in the Carina arm at a distance of $12 \mathrm{kpc}$. The $l-b$ CO map of G303.90.4 (integrated over velocities in the 7 to $50 \mathrm{~km} \mathrm{~s}^{-1}$ interval) can be found in Grabelsky et al. (1988). Two adjacent $\mathrm{CO}$ maxima are visible, with the $\mathrm{TeV}$ source located on one of them, giving a $\mathrm{TeV}$ source averaged $\mathrm{CO}$ intensity of $\langle W(\mathrm{CO})\rangle \sim 8 \mathrm{~K} \mathrm{~km} \mathrm{~s}^{-1}$. The target density at the contours corresponding to the $\mathrm{TeV}$ maximum is then $n \sim 10$ to $20 \mathrm{~cm}^{-3}$, if a cloud scale size corresponding to the FWHM of the integrated temperatures on the image is assumed. The required energy in cosmic rays is now $E_{\mathrm{CR}} \sim 3 \times 10^{50} \mathrm{erg}$. Such energetics would require a relative powerful supernova explosion and it remains to be shown if an old SNR shell with 40 pc radius can still support cosmic ray production at the required level.

An interesting counterpart for HESS J1303-631 in connection with $\mathrm{CO}$ solution (ii) may be the $\mathrm{OB}$ stellar association Cen OB1. Via the stellar winds of their member stars, OB associations are thought to supply considerable kinetic energy into the surrounding media. Cen OB1 contains 20 stars of spectral type 09 , suggesting that some member stars may have evolved beyond the supernova stage. The Wolf-Rayet star $\theta$ Mus is also a member and may be a triple ( $\mathrm{WR}+\mathrm{O}+\mathrm{O}$ stars) system (Hartkopf et al. 1999). McClure-Griffiths et al. (2001) identify the H I shell GSH 305+01-24 as being blown out by the OB association Cen OB1. The estimated distance of GSH $305+01-24$ at $2.2 \mathrm{kpc}$ is consistent with that of Cen OB1 (2.5 kpc, Humphreys 1978). Moreover, the estimated kinetic

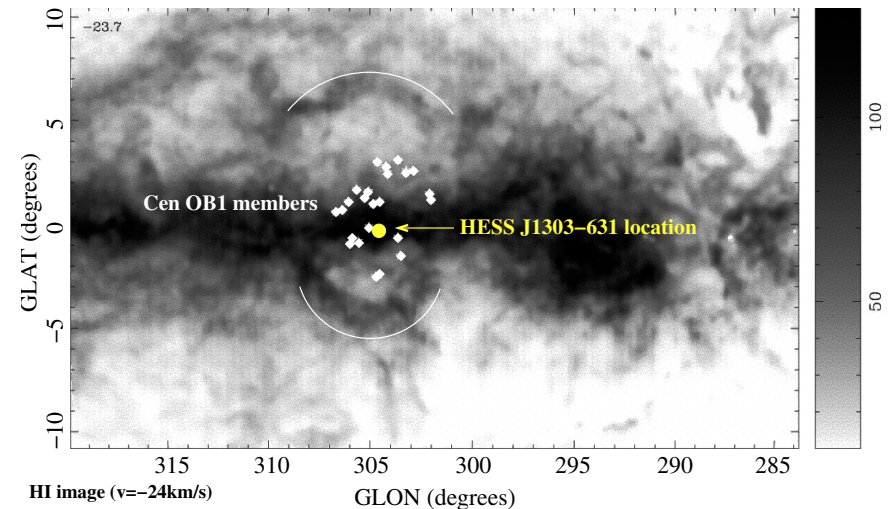

Fig. 7. H I image $\left(\sim 35^{\circ} \times 20^{\circ}\right)$ at $v=-24 \mathrm{~km} \mathrm{~s}^{-1}$ of the shell GSH 305+01-24 (McClure-Griffiths et al. 2001). The member stars of Cen OB1, location of HESS J1303-631 and approximate outer boundaries of GSH 305+01-24 are indicated.

energy required to maintain the $\mathrm{HI}$ shell expansion at $\sim 10 \times$ $10^{51} \mathrm{erg}$ is met by that of Cen OB 1 at $\sim 8 \times 10^{51} \mathrm{erg}$, based on stellar wind luminosities and ages of member stars. Figure $7 \mathrm{de}-$ picts the positional relationship between the $\mathrm{H}$ I shell, Cen OB1 member stars and HESS J1303-631. Assuming a canonical factor 0.1 for kinetic energy to comsic-ray energy conversion, $\sim 8 \times 10^{50}$ erg would be available for cosmic-ray production. This link between a $\mathrm{TeV} \gamma$-ray source and an $\mathrm{OB}$ association mirrors very closely that of the first unidentified $\mathrm{TeV}$ source TeV J2032+4130 and Cygnus OB2 discovered by the HEGRA collaboration (Aharonian et al. 2002a, 2004a).

Note, that the distances of Cen OB1 and GSH 305+01-24 coincide with the first kinematic CO solution of $2.1 \mathrm{kpc}$ (within uncertainties). A number of scenarios discussed below could give rise to the $\mathrm{TeV}$ emission:

(a) The stellar winds from member stars of Cen OB1 provide conditions for the acceleration of cosmic rays, perhaps via shocks setup at the interfaces of the various stellar winds. These cosmic rays in turn could illuminate a nearby molecular cloud. If the average distance of HESS J1303-631 to the Cen OB 1 member stars is of order $\leq 2^{\circ}$, the required energy to account for the $\mathrm{TeV}$ source, assuming all member stars contribute, would be $4 \times 10^{48}(2.0 / 0.32)^{2} \mathrm{erg}=$ $2 \times 10^{50} \mathrm{erg}$. This is consistent with the cosmic-ray energetics of Cen OB1 discussed earlier. Here, a source radius of $0.32^{\circ}$ ( $95 \%$ or $2 \sigma$ containment radius) is assumed. A dense cloud situated at the location of HESS J1303-631, could therefore be a valid solution. The energetics could also be met by a single or a few member stars, provided they were close enough. The CO data however, also suggest the presence of numerous dense clouds in the vicinity of Cen OB1. The fact that $\mathrm{TeV}$ emission from just one location is seen could imply that HESS J1303-631 is somehow special in relation to the distance to the local cosmic-ray accelerator, and its geometry. Detailed modeling of the acceleration and diffusion of cosmic-rays in this region will be needed to verify this scenario.

(b) As for situation (a) but the $\mathrm{TeV}$ emission may arise from a leptonic process such as inverse-Compton upscattering 
of local soft photons. The localised nature of HESS J1303631 would then require some extra conditions namely: the soft photon distribution (which may be dominated by UV photons from the member stars) is also localised; the acceleration and diffusion properties of electrons is such that the energetic electrons are confined.

(c) If the oldest member stars already evolved into the supernova stage, the SNOB scenario of Montmerle (1979) may also explain the existence of HESS J1303-631, but it remains then to be shown why this SNR is not seen at X-ray and radio wavelengths.

\subsection{Clumps of annihilating dark matter}

A possible source of $\mathrm{TeV} \gamma$-ray emission could also be a clump of annihilating dark matter particles (Moore et al. 1999) which would result in an energy spectrum with a cut-off at the mass of the dark matter particle. The galactic halo could contain a huge amount of such clumps comprised e.g. of neutralinos.

Although not very likely, it is possible to reproduce in Monte Carlo simulations the observed flux with the annihilation radiation from high mass neutralinos, but it is not possible to reproduce the luminal profile measured from HESS J1303-631 assuming a Navarro-Frenk-White (NFW) dark matter density profile (Navarro et al. 1996). For the NFW and other commonly used profiles a narrower luminal profile is expected than obtained from HESS J1303-631.

\section{Summary and conclusions}

A new unidentified $\mathrm{TeV} \gamma$-ray source HESS J1303-631 was serendipitously discovered in a dataset which was initially taken on the binary system PSR B1259-63/SS 2883 which was also discovered at $\mathrm{TeV}$ energies (see a parallel paper: Aharonian et al. 2004c). For the first time in $\mathrm{TeV} \gamma$-ray astronomy, the detection and analysis of two sources within the same field of view is achieved which subsequently also occured in other pointings towards the galactic plane, showing the potential of the new generation of ground-based experiments - such as HESS - with the stereoscopic observation mode and its large field of view of $\sim 5^{\circ}$.

HESS J1303-631 was found to be clearly extended with a width of an assumed intrinsic Gaussian emission profile of $\sigma=(0.16 \pm 0.02)^{\circ}$. The energy spectrum can be described by a power-law with a photon index of $\Gamma=2.44 \pm 0.05_{\text {stat }} \pm 0.2_{\text {syst }}$. The integral flux above $380 \mathrm{GeV}$ was found to remain on a constant level of $(17 \pm 3) \%$ of the flux from the Crab Nebula during the observations taken between February and June, 2004. Detailed studies on morphology and spatially-resolved energy spectra are underway.

Up to now, no counterpart at other wavelengths was identified. However, the location close to the galactic plane places HESS J1303-631 in the vicinity of a variety of possible objects which might be involved in the production mechanisms explaining the observed $\mathrm{TeV} \gamma$-ray emission. A pulsar wind nebula powered by the nearby pulsar PSR J1301-6305 is one of the interesting candidates. Another possibility would be the Cen OB1 stellar association at a distance of $2.5 \mathrm{kpc}$ which provides enough kinetic energy by stellar winds. The interaction of an expanding supernova remnant shell with a molecular cloud as target material can also be seen as an interesting configuration for $\mathrm{TeV} \gamma$-ray production. Three molecular clouds are located along the line of sight of HESS J1303-631: The Coalsack nebula at a distance of $\sim 175 \mathrm{pc}$, a cloud in the Carina-Sagittarius arm at $2.1 \mathrm{kpc}$, resp. $7.7 \mathrm{kpc}$, and the giant molecular cloud GMC G303.0-0.4 in the Carina Arm at a distance of $12 \mathrm{kpc}$. Finally, the possibility of a clump of annihilating dark matter particles was considered, which however can be excluded since the luminal profile of HESS J1303-631 is not reproduced.

In summary, no clear counterpart was identified to date. Therefore HESS J1303-631 has to be considered as the second unidentified TeV source detected, following TEV J2032+4130 discovered in the Cygnus region by HEGRA (Aharonian et al. 2002a, 2004a). Meanwhile, the results of the HESS galactic plane scan revealed more unidentified $\mathrm{TeV} \gamma$-ray sources (Aharonian et al. 2005b), thus further opening the door to a new class of (yet-unidentified) $\mathrm{TeV} \gamma$-ray sources. To further investigate possible production mechanisms and to understand this new region of the non-thermal universe future multiwavelength observations (especially in X-rays) are essential and partially already initiated (Mukherjee \& Halpern 2005).

Acknowledgements. The support of the Namibian authorities and of the University of Namibia in facilitating the construction and operation of HESS is gratefully acknowledged, as is the support by the German Ministry for Education and Research (BMBF), the Max Planck Society, the French Ministry for Research, the CNRS-IN2P3 and the Astroparticle Interdisciplinary Programme of the CNRS, the UK Particle Physics and Astronomy Research Council (PPARC), the IPNP of the Charles University, the South African Department of Science and Technology and National Research Foundation, and by the University of Namibia. We appreciate the excellent work of the technical support staff in Berlin, Durham, Hamburg, Heidelberg, Palaiseau, Paris, Saclay, and in Namibia in the construction and operation of the equipment. This research has made use of the SIMBAD database, operated at CDS, Strasbourg, France.

\section{References}

Aharonian, F. A. 1990, Ap\&SS, 180, 305

Aharonian, F. A., Drury, L. O'C., \& Völk, H. J. 1994, A\&A, 285, 645

Aharonian, F. A., Atoyan, A. M., \& Kifune, T. 1997, MNRAS, 291, 162

Aharonian, F., Akhperjanian, A., Beilicke, M., et al. (HEGRA collab.) 2002a, A\&A, 393, L37

Aharonian, F. A., Akhperjanian, A. G., Beilicke, M., et al. (HEGRA collab.) 2002b, A\&A, 395, 803

Aharonian, F., et al. (HEGRA collab.) 2004a, A\&A, in press [arXiv: astro-ph/0501667]

Aharonian, F., Akhperjanian, A. G., Aye, K.-M., et al. (HESS collab.) 2004b, Astropart. Phys., 22, 109

Aharonian, F., et al. (HESS collab.) 2004c, A\&A, submitted

Aharonian, F., Akhperjanian, A. G., Aye, K.-M., et al. (HESS collab.) 2005a, A\&A, 430, 865

Aharonian, F., Akhperjanian, A. G., Aye, K.-M., et al. (HESS collab.) 2005b, Science, 307, 1938 
Beilicke, M., Ouchrif, M., Rowell, G., \& Schlenker, S. (HESS collab.) 2004, IAU Circular \#8300

Beilicke, M., Khelifi, B., Masterson, C., et al. (HESS collab.) 2005, High Energy Gamma-Ray Astronomy, AIP Conf. Proc., 745, 347

Bernlöhr, K., Carrol, O., Cornils, R., et al. (HESS collab.) 2003, Astropart. Phys., 20, 111

Bock, D. C.-J., Large, M. I., \& Sadler, E. M. 1999, AJ, 117, 1578

Cornils, R., Gillessen, S., Jung, I., et al. (HESS collab.) 2003, Astropart. Phys., 20, 129

Dame, T. M., Koper, E., Israel, F. P., \& Thaddeus, P. 1993, ApJ, 418, 730

Drury, L. O’C., Aharonian, F. A., \& Völk, H. J. 1994, A\&A, 287, 959

Funk, S., Hermann, G., Hinton, J., et al. (HESS collab.) 2004, Astropart. Phys., 22, 285

Grabelsky, D. A., Cohen, R. S., Bronfman, L., \& Thaddeus, P. 1988, ApJ, 331, 181

Bronfman, L., Alvarez, H., Cohen, R. S., \& Thaddeus, P. 1989, ApJS, 71,481

Green, D. A. 2004, Bull. Astron. Soc. India, 32, 335

Hartkopf, W. I., Mason, B. D., Gies, D. R., et al. 1999, AJ, 118, 509

Hartman, R. C., Bertsch, D. L., Bloom, S. D., et al. 1999, ApJS, 123, 79

Hinton, J. A. (HESS collab.) 2004, New Astron. Rev., 48, 331

Humphreys, R. M. 1978, ApJS, 38, 309

Kaaret, P., \& Cottam, J. 1996, ApJ, 462, L35

Li, T., \& Ma, Y. 1983, ApJ, 272, 317
Manchester, R. N., Hobbs, G. B., Teoh, A., \& Hobbs, M. 2005, AJ, in press [arXiv: astro-ph/0412641]

McClure-Griffiths, N., Dickey, J. M., Gaensler, B. M., \& Green, A. J. 2001, ApJ, 562, 424

Montmerle, T. 1979, ApJ, 231, 95

Moore, B., Ghigna, S., \& Governato, F. 1999, ApJ, 524, L19

Mukherjee, R., \& Halpern, J. P. 2005, ApJ, submitted

Naurois, M., de Guy, J., Djannati-Ataï, A., \& Tavernet, J.-P. (HESS collab.) 2003, Proc. 28th ICRC (Tsukuba) (Tokyo: Univ. Academy Press ), 2907

Navarro, J. F., Frenk, C. S., \& White, S. D. M. 1996, ApJ, 462, 563

Navarro, J. F., Frenk, C. S., \& White, S. D. M. 1997, ApJ, 490, 493

Nyman, L.-Å., Bronfman, L., \& Thaddeus, P. 1989, A\&A, 216, 185

Ochsenbein, F., Bauer, P., \& Marcout, J. 2000, A\&AS, 143, 221

Paladini, R., Burigana, C., Davies, R. D., et al. 2003, A\&A, 397, 213

Strong, A. W., Bloemen, J. B. G. M., Dame, T. M., et al. 1988, A\&A, 207,1

Rowell, G. 2003, A\&A, 410, 389

van der Swaluw, E., Achterberg, A., Gallant, Y. A., \& Tóth, G. 2001, A\&A, 380, 309

Vincent, P., Denance, J.-P., Huppert, J.-F., et al. (HESS collab.) 2003, Proc. of the 28th ICRC (Tsukuba), 2887

Wouterloot, J. G. A., \& Brand, J. 1989, A\&AS, 80, 149

Wright, A. E., Griffith, M. R., Burke, B. F., \& Ekers, R. D. 1994, ApJS, 91,111 